\title{
Colloidal Monolayer Trapped near a Charged Wall: A Synchrotron X-Ray Diffraction Study
}

\author{
D. K. Satapathy, ${ }^{1, *}$ O. Bunk, ${ }^{1}$ K. Jefimovs,${ }^{1}$ K. Nygård,${ }^{1}$ H. Guo, ${ }^{2}$ A. Diaz, ${ }^{1}$ E. Perret, ${ }^{1}$ F. Pfeiffer,${ }^{1,3}$ C. David, ${ }^{1}$ \\ G. H. Wegdam, ${ }^{2}$ and J.F. van der Veen ${ }^{1,4}$ \\ ${ }^{1}$ Research Department of Synchrotron Radiation and Nanotechnology, Paul Scherrer Institut, 5232 Villigen PSI, Switzerland \\ ${ }^{2}$ Van der Waals-Zeeman Instituut, Universiteit van Amsterdam, 1018 XE Amsterdam, The Netherlands \\ ${ }^{3}$ École Polytechnique Fédérale de Lausanne, 1015 Lausanne, Switzerland \\ ${ }^{4}$ ETH Zürich, 8093 Zürich, Switzerland \\ (Received 26 June 2008; published 24 September 2008)
}

\begin{abstract}
Using x-ray diffraction from microfluidic channel arrays, we have determined concentration profiles of charge-stabilized silica colloids (radius $60 \pm 2 \mathrm{~nm}$ ) confined between two like-charged dielectric walls at a few hundred nanometer distance. In solutions of very low ionic strength, strongly repulsive Coulomb interactions drive the colloids toward the central region between the walls. The addition of a small quantity of salt ions $(0.2 \mathrm{mM})$ causes a dense colloidal monolayer to be trapped near the walls.
\end{abstract}

DOI: 10.1103/PhysRevLett.101.136103

Colloidal dispersions display physical properties different from the bulk if they are confined in narrow slits or pores. It is of great interest to understand how the confined geometry modifies the interactions between the colloidal particles. A charge-stabilized colloidal suspension between two flat like-charged walls at variable distance serves here as a model, because this system is well-defined and the strength and range of the repulsive Coulombic colloid-colloid $(c-c)$ and colloid-wall $(c-w)$ interactions are tunable [1]. For example, one may enhance the screening of the Coulomb interactions well beyond that caused by the colloid's counterions by adding salt ions to the dispersion. The $c-c$ and $c-w$ interactions determine the concentration profile of colloids across the confining gap as well as the colloid's pair distribution $g\left(r_{\|}\right)$within the plane of confinement. A series of video microscopy measurements of $g\left(r_{\|}\right)$have provided evidence for the existence of a longrange attractive interaction between two colloids in the presence of a wall [2], which is a remarkable result, given their strong Coulomb repulsion in the bulk. Theoretical mean-field descriptions on colloid interactions which are based on either the use of Derjaguin-Landau-VerweyOverbeek potentials or solving the nonlinear PoissonBoltzmann equation using appropriate boundary conditions have brought confinement effects into evidence [35]. In parallel, models which explicitly account for the discrete nature of the solvent or for the presence of possible correlations in the density distribution of the counterions surrounding the colloids ("macroions") and the charged walls provide evidence for the existence of short-range attractive forces in the neighborhood of the walls [6-9]. For computational reasons, simulations have taken interactions between only a very small number of ions into account. Recent microscopy studies have reported the emergence of a long-range attractive force near a single wall upon increasing the ionic strength of the solution [10]. Further investigation of the nature and range of these interactions and the role of ionic strength is warranted.
PACS numbers: 68.08. $-\mathrm{p}$, 61.05.C - , 82.70.Dd, 87.19.rh

In this Letter, we report the trapping of a colloidal monolayer near the confining walls. Since it occurs only for a specific range of ionic strengths of the solution, the condensation of colloidal particles into a monolayer results from major changes in the electrostatic screening near the walls. The trapped monolayers cause the confinement channel to become narrower by twice the particle diameter, an effect of direct relevance to diffusion of complex fluids through narrow pores.

Using synchrotron x-ray diffraction, we have determined the concentration profile of silica colloids across confining channels of different widths. The ionic strength of the fluid and thereby the range of Coulomb interactions between the macroions and between the macroions and the charged channel walls were tuned in situ by adding a 1:1 electrolyte $(\mathrm{LiCl})$. In order to enhance the diffraction signal, microfluidic arrays of identical channels were employed [11]. The array acts as a phase grating, diffracting the x-ray beam into many orders [12]. From the measured efficiencies of the diffraction orders, the concentration profile of the colloids is uniquely derived [13]. Our method differs from optical video microscopy $[2,10]$ in several respects: (i) It directly measures laterally averaged density distributions across the channels instead of the positions of individual particles; (ii) it yields an ensemble average over an array of channels of equal width instead of a single realization; (iii) it enables an accurate determination of the distribution of the colloids' positions relative to the walls; (iv) its much shorter wavelength, in principle, allows for investigations of complex fluids and channels down to much smaller length scales; (v) it does not require any optical contrast between the colloids and dispersing medium.

The colloidal dispersions consist of 10,15 , or $20 \mathrm{vol} \%$ silica spheres having a radius $r_{m}=60 \pm 2 \mathrm{~nm}$ in a solvent of 55\% benzyl alcohol and $45 \%$ ethanol [14]. The silanol $(\mathrm{SiOH})$ groups on the colloid's surface dissociate in the solvent producing a negative surface charge density of 
typically $5 \times 10^{-4} \mathrm{C} \mathrm{m}^{-2}[15,16]$ and releasing $\mathrm{H}^{+}$counterions into the solution. The silicon walls of the confining channels are covered by a thin natural oxide and are likewise negatively charged and also release counterions. The bulk Debye screening length is given by $\kappa^{-1}=$ $\sqrt{1 /\left[4 \pi \lambda_{b}\left(Z \bar{n}+2 n_{s}\right)\right]}$, where $Z$ is the number of charges per colloid, $Z \bar{n}$ is the number density of counterions, and $n_{s}$ is the number density of the monovalent electrolyte added to the dispersion. The Bjerrum length $\lambda_{b}=e^{2} / 4 \pi \epsilon_{0} \epsilon_{s} k_{B} T$ equals $3.1 \mathrm{~nm}$, with the solvent's relative dielectric constant $\epsilon_{s}=18.3, k_{B}$ Boltzmann's constant, and the temperature $T=300 \mathrm{~K}$. For a $10 \mathrm{vol} \%$ base solution (fluid I), $\kappa^{-1} \simeq 35 \mathrm{~nm}$. After addition of $0.2 \mathrm{mM} \mathrm{LiCl}$ (fluid II), the bulk screening length reduces to $\kappa^{-1} \simeq 10 \mathrm{~nm}$, a value significantly smaller than the colloid radius. Confinement is expected to give rise to important changes in the screening behavior, which may be expressed by a positiondependent screening length that is smaller near the walls. An important parameter here is the strength of the Coulomb coupling between the macroions and the counterions as expressed by the coupling parameter $\Gamma_{m c}=$ $Z \lambda_{b} /\left(r_{m}+r_{c}\right)$, with $r_{c}$ being the radius of the counterion. We find $\Gamma_{m c} \simeq 8$, so we may expect condensation of neutralizing counterions onto both the colloid surfaces and the walls.

A schematic of our experimental method is shown in Fig. 1. The dispersion was injected into a microfluidic chip containing different periodic arrays of rectangular channels [11]. A single chip contains 50 arrays, each having 600 periods of the same length but a different channel width. The period $p$ equals $1005 \mathrm{~nm}$, and the channel width $w$ ranges from 200 to $620 \mathrm{~nm}$ at an average increment of $9 \mathrm{~nm}$. The height $h$ of the channels is $4 \mu \mathrm{m}$. The microfluidic chip is covered by a glass plate to prevent evaporation of the fluid. The gap between the glass plate

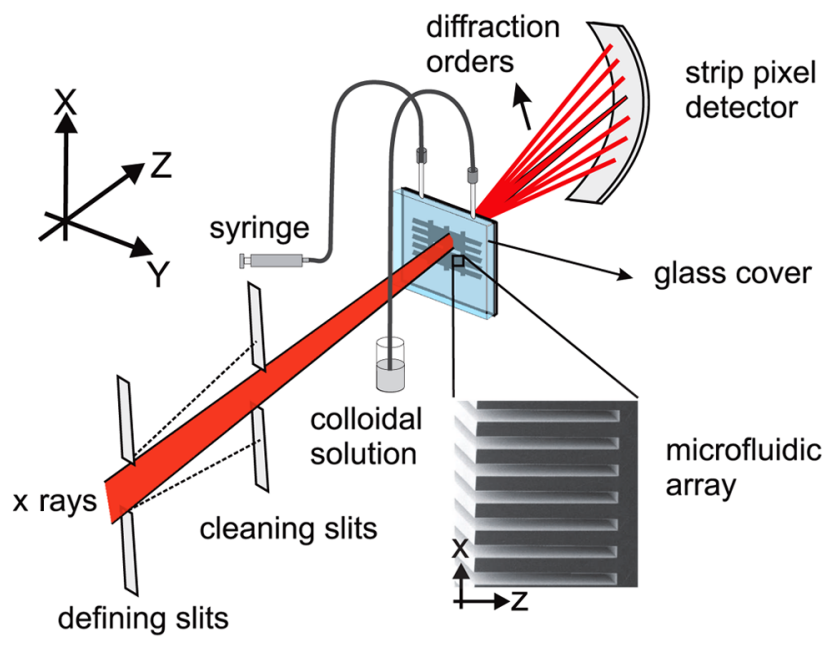

FIG. 1 (color online). Experimental setup and scattering geometry. The x-ray beam impinges under normal incidence onto the microfluidic chip on which several arrays of channels were prepared. and the array acts as a reservoir of fluid allowing a free exchange of colloids and microions. An x-ray beam of wavelength $\lambda=0.104 \mathrm{~nm}$ was directed onto each of the periodic arrays on the chip, and their diffraction patterns were measured in transmission geometry using a microstrip detector (strip pitch $50 \mu \mathrm{m}$ ) positioned 5.03 meters behind the sample. The momentum resolution is $6 \times$ $10^{-4} \mathrm{~nm}^{-1}$. From each array, typically 80 diffraction orders were measured. Figure 2 shows as an example the orders measured from an array containing fluid I and from an identical array containing fluid II (because of symmetry, only the positive orders are displayed).

The large momentum range $\Delta q=0.5 \mathrm{~nm}^{-1}$ corresponds to a spatial resolution of $\Delta x=2 \pi / \Delta q=$ $12.5 \mathrm{~nm}$, and the spacing of $6.25 \times 10^{-3} \mathrm{~nm}^{-1}$ between the diffraction peaks corresponds with the known array period of $1005 \mathrm{~nm}$. The inset in Fig. 2 highlights the differences between the intensities of the orders for fluids I and II. The structural rearrangements caused by the addition of a small quantity of electrolyte $(0.2 \mathrm{mM})$ are quantified as follows. The efficiencies of all measured diffraction orders are extracted from the data by peak integration. The difference $\Delta \phi(x)$ between the phases of diffracted exiting waves across the channel for fluids II and $\mathrm{I}$ is then determined from the Fourier series [17]

$$
\Delta \phi(x) \simeq \sum_{m=-M}^{M} \frac{\left(\eta_{m}^{\mathrm{II}}-\eta_{m}^{\mathrm{I}}\right)}{2 F_{m} \sin \Phi} \cos (2 \pi m x / p),
$$

where $\left\{\eta_{m}^{\mathrm{I}}\right\}$ and $\left\{\eta_{m}^{\mathrm{II}}\right\}$ are the sets of diffraction efficiencies measured for fluids I and II, respectively, $M$ is the number of positive orders, $F_{m}=\sin (\pi m w / p) /(\pi m)$ is the form factor of a single rectangular channel period of unit height, $\Phi=2 \pi h \Delta \delta / \lambda$ is the average difference in the phase of the exiting wave between the bottom and the top of the channel, and $\Delta \delta$ is the jump in the real part $\delta$ of the average $\mathrm{x}$-ray refractive index $n=1-\delta-i \beta$ across the silicon-fluid interface. A direct inversion of the diffraction pattern as in Eq. (1) is possible if the density modulations

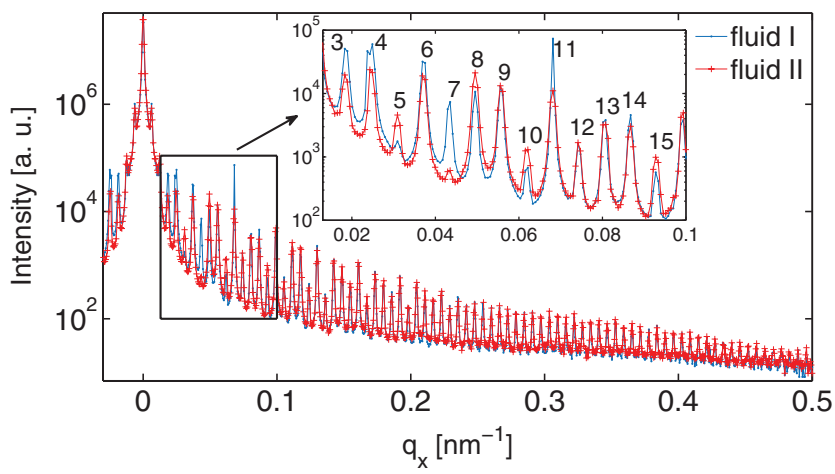

FIG. 2 (color). Measured diffraction patterns from identical microfluidic arrays containing the colloidal dispersion with no salt ions added (fluid I, blue curve) and after addition of $0.2 \mathrm{mM}$ of salt ions (fluid II, red curve). The inset shows a magnified view of the 3 rd-15th diffraction orders. 


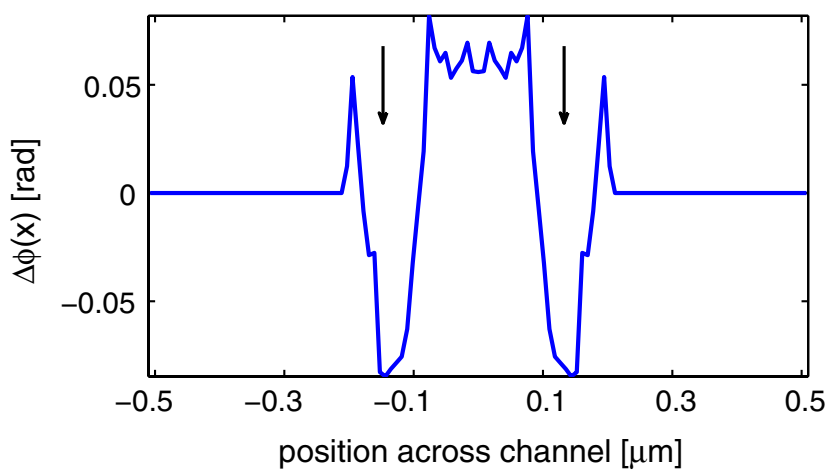

FIG. 3 (color online). Difference between the phase profiles of diffracted outgoing waves for fluids II and I across a channel of $407 \mathrm{~nm}$ width. The arrows indicate the position of monolayers in the channel.

in the fluid represent a weak phase object for all diffraction orders considered. Applying Eq. (1) to the diffraction orders shown in Fig. 2, we find that in fluid II a dense colloid monolayer has formed near the walls (see Fig. 3 for a channel width $w=407 \mathrm{~nm}$ ). The phase profiles extracted from Eq. (1) are not corrected for refraction of the x-ray beam from the channel walls. One may account for this by replacing the kinematic form factor $F_{m}$ by its dynamical counterpart. Instead, we have employed the microfluidic array phase-profiling (MAPP) technique of Ref. [13]. It is based on a phase retrieval algorithm which allows the phase and amplitude of the exit wave field to be determined under certain additional constraints. Back propagation of the wave then eliminates refraction effects from the channel walls [18]. Our structural analyses of

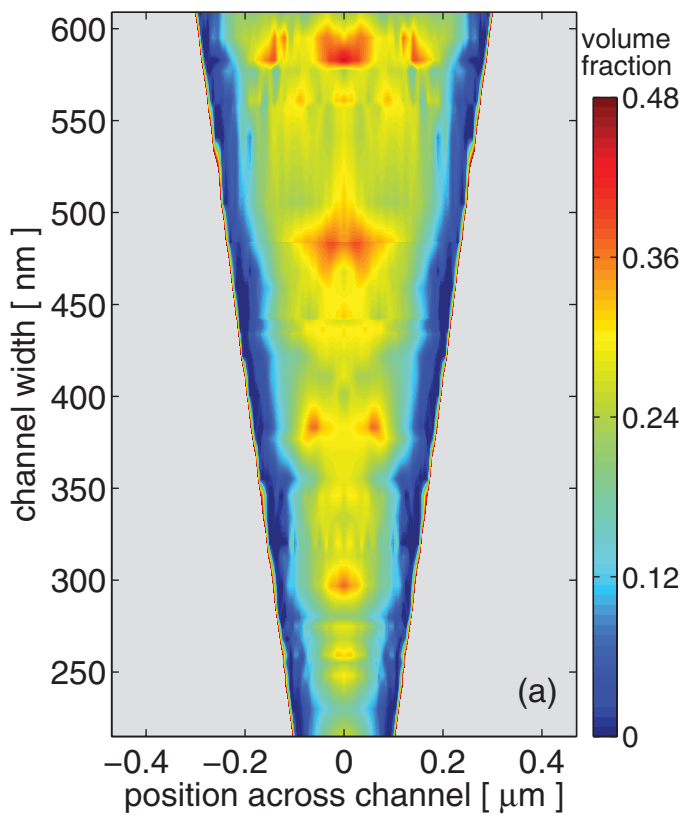

fluids I and II based on MAPP are summarized in Fig. 4, which shows the measured concentration profiles as a function of channel width $w$ expressed in local volume fractions.

We first discuss the results for the dispersion with 10 vol \% colloid without added salt (fluid I). Figure 4(a) shows that this dispersion has its maximum colloid concentration near the center of the channel. This indicates a strong repulsion from like-charged walls. In addition, pronounced layering oscillations are present with maximum concentrations, e.g., at 380 and $590 \mathrm{~nm}$ where two and three layers have formed.

Dispersions with $0.2 \mathrm{mM}$ of $\mathrm{LiCl}$ added (fluid II) are found to have a markedly different concentration profile within the channel; see Fig. 4(b). A segregated monolayer is present at both walls, irrespective of the channel width. This behavior has been reproduced in five different measurements and for three different volume percentages $10 \%$, $15 \%$, and $20 \%$ of stock fluid. The pronounced monolayer disappears upon increasing the concentration of the electrolyte from $0.2 \mathrm{mM}$ to $0.2 \mathrm{M}$ [19]. This shows that the monolayer formation is related to subtle changes in the electrostatic screening near the walls, not to van der Waals attraction. It also appears that the walls act independently of each other in trapping the colloids.

In Fig. 4(b), layering oscillations are observed in the space between the trapped monolayers (see channel widths of 395 and $523 \mathrm{~nm}$ for one- and two-layer structures). The maxima in the concentration of centrally located colloids appear at channel widths $w$ different from those for fluid I. Our measurements show that the monolayers make the channel effectively smaller and act as new repulsive walls

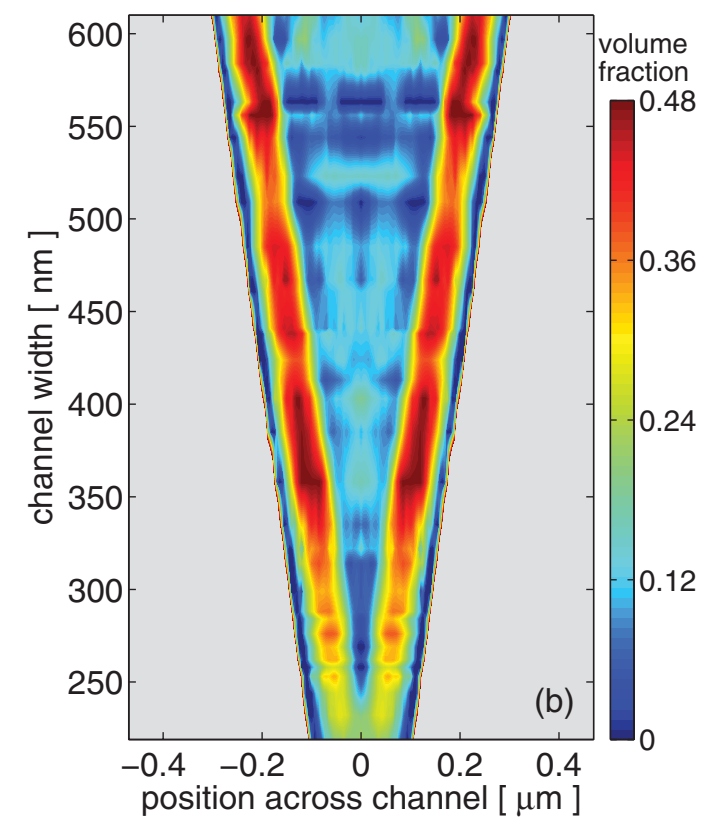

FIG. 4 (color). Contour plots of the local volume fraction of colloids across the confining channel as a function of the cavity width $w$ : (left) for the dispersion without salt added (fluid I) and (right) for the dispersion after addition of $0.2 \mathrm{mM}$ of salt ions (fluid II). The gray outer region is the area of the confining silicon walls. 
for the remaining colloids. We believe that these observations are consistent with a model in which the effective screening length $\kappa^{-1}(x)$ is position-dependent [9]. As viewed from a single colloid in the monolayer, $\kappa^{-1}(x)$ would be smaller at the side facing the walls than at the side facing the center of the channel. This may be explained as follows. A moderate Coulomb coupling between macro- and microions (here, $\Gamma_{m c} \simeq 8$ ), results in a nonnegligible condensation of counterions onto the walls and colloid surfaces [20]. Correlations in the spatial distributions of these counterions may then enhance the concentration of counterions in the narrow space between a colloid and the wall. Apart from reducing the local screening length, this effectively leads to an attractive force. The effect of correlations is further enhanced for two neighboring colloids instead of one [9], which explains our observation of a dense monolayer. We note, however, that the measured trapping effect is the net result of all forces acting on the colloids. These should include a repulsive force directed away from the wall which is associated with an image charge behind the walls of the same sign $\left(\epsilon_{s}>\right.$ $\left.\epsilon_{\text {wall }}\right)$ [5] and a repulsive force directed towards the wall which is exerted by the colloidal particles near the midplane of the channel. Our findings are consistent with previous predictions of adsorbed monolayer formation in confinement which explicitly account for correlated $c-c$ and $c-w$ interactions [3,21]. However, the effect of salt ion addition was not considered in these studies.

The addition of a small quantity of salt $(0.2 \mathrm{mM})$ creates the condition of a net attractive force towards the walls by increasing the number of counterions near the walls and lowering the screening length so that colloids can explore the regions very close to the charged walls. By contrast, in the dispersion without salt, the strong repulsive force between walls and colloids does not allow them to explore the wall regions. The colloids then stay sufficiently far away from the walls, and the screening length varies much less over the distance of a colloid diameter. At the other extreme, if a large quantity of salt $(0.2 \mathrm{M})$ is present, the colloids explore the entire channel width, but the screening length has decreased by so much $\left(\kappa^{-1} \simeq 0.3 \mathrm{~nm}\right)$ that it, again, varies little around the surface of a single colloid. It appears therefore that the trapping of the colloidal particles near the walls is caused by a subtle shift in the balance of forces acting on the colloids, which occurs only for a certain range of intermediate salt ion concentrations.

In conclusion, we have quantitatively determined laterally averaged concentration profiles of charge-stabilized colloids in narrow charged channels and have observed the trapping of a monolayer of colloid near the channel walls upon addition of a small quantity of salt ions. We conjecture that this effect is related to a strongly reduced electrostatic screening length near the walls and the occurrence of spatial correlation effects in the distribution of neutralizing counterions. For the colloidal system investigated, the trapping occurs within a specific range of ionic strengths of the solvent. A full understanding of this intriguing phenomenon requires further investigations of systems with different charges on colloids and walls, valencies of the salt ions, and dielectric constants of solvent and walls.

The diffraction experiments were performed at beam line X04SA of the Swiss Light Source, Paul Scherrer Institut, Villigen, Switzerland. We thank the beam line staff for assistance. We gratefully acknowledge the assistance of M. Dierolf for the experiments.

*dillip.satapathy@psi.ch

[1] J. N. Israelachvili, Intermolecular and Surface Forces (Academic, New York, 1991), 2nd ed.

[2] D. G. Grier and Y. Han, J. Phys. Condens. Matter 16, S4145 (2004).

[3] P. González-Mozuelos and M. Medina-Noyola, J. Chem. Phys. 94, 1480 (1991).

[4] P. González-Mozuelos and M. Medina-Noyola, J. Chem. Phys. 93, 2109 (1990).

[5] H. H. von Grünberg and E. C. Mbamala, J. Phys. Condens. Matter 13, 4801 (2001).

[6] D. Goulding and J.-P. Hansen, Europhys. Lett. 46, 407 (1999).

[7] A.D. Trokhymchuck, D. Henderson, D. T. Wasan, and A. Nikolov, Ionic Soft Matter: Modern Trends in Theory and Applications (Springer, New York, 2005).

[8] J. Chakrabarti and H. Löwen, Phys. Rev. E 58, 3400 (1998).

[9] E. Allahyarov, I. D’Amico, and H. Löwen, Phys. Rev. E 60, 3199 (1999).

[10] M. Polin, D. G. Grier, and Y. Han, Phys. Rev. E 76, 041406 (2007).

[11] A. Diaz, C. David, H. Guo, H. Keymeulen, F. Pfeiffer, G. Wegdam, T. Weitkamp, and J. F. van der Veen, Physica (Amsterdam) 357B, 199 (2005).

[12] A. Diaz and J. F. van der Veen, Thin Solid Films 515, 5645 (2007).

[13] O. Bunk, A. Diaz, F. Pfeiffer, C. David, D. K. Satapathy, and J.F. van der Veen, Acta Crystallogr. Sect. A 63, 306 (2007).

[14] The optical refractive index of this solvent matches that of the colloids, which minimizes the van der Waals attraction between the colloids.

[15] D. O. Riese, Ph.D. thesis, University of Amsterdam, 2000.

[16] M. Raşa and A.P. Philipse, Nature (London) 429, 857 (2004).

[17] K. Nygård et al. (to be published).

[18] K. Nygård, D. K. Satapathy, O. Bunk, F. Pfeiffer, C. David, and J.F. van der Veen, Appl. Phys. Lett. 92, 214105 (2008).

[19] O. Bunk et al., Phys. Rev. E 75, 021501 (2007).

[20] P. Linse, J. Phys. Condens. Matter 14, 13449 (2002).

[21] J. J. Liétor-Santos, M. Chávez-Páez, M. Márquez, A. Fernández-Nieves, and M. Medina-Noyola, Phys. Rev. E 76, 050403 (2007). 